Revista de Psicología Vol. 36 (1), 2018 (ISSN 0254-9247)

\title{
Dynamique identitaire et projet
}

\author{
Anne-Marie Costalat-Founeau ${ }^{1}$, Audrey Gosset ${ }^{2}$ \\ Université Paul Valéry Montpellier - France, Laboratoire Epsylon EA4556
}

L'objectif de cet article est de présenter les processus qui animent la dynamique identitaire phasique de l'identité sociale permettant de définir simultanément une représentation de soi et du projet personnel et professionnel. De ce fait, elle implique des niveaux d'ancrages différenciés dans les organisations, les groupes et la société qui contribuent à façonner le projet du soi. Nous illustrerons ceci, grâce à des cas et des situations en lien avec le contexte, la mémoire autobiographique mais aussi les émotions, le sentiment de capacité et la motivation. Une de nos orientations stipule que l'identité sociale s'organise dans une dynamique représentationnelle où les phases d'action deviennent des facilitateurs de la construction identitaire et laissant des formes dans la mémoire expérientielle nécessaires à la construction du projet.

Mots clés: Dynamique identitaire, projet, action, orientation.

\section{Dinámica de la identidad y proyecto}

El objetivo de este artículo es presentar los procesos que impulsan la dinámica de identidades fásicas de la identidad social, permitiendo definir simultáneamente una representación del self, del proyecto personal y profesional. Este eje supone diferentes niveles de anclajes en las organizaciones, los grupos y la sociedad los cuales contribuyen a dar forma al proyecto del si mismo. Ilustramos este paradigma a travès los casos y situaciones relacionadas con el contexto, la memoria autobiográfica, pero también las emociones, el sentimiento de capacidad y la motivación. Nuestra orientación estipula que la identidad social se organiza en una dinámica representacional donde las fases de la acción se convierten en facilitadores de la construcción de la identidad y dejan algunas formas en la memoria experiencial necesarios para la construcción del proyecto.

Palabras clave: Dinámica de la identidad, proyecto, acción, orientación.

$1 \quad \mathrm{PhD}$ en Psychologie sociale. Professeur de Psychologie sociale et du travail à l'université Paul Valéry Montpellier 3. Adresse postale: Univ Paul Valéry Montpellier 3. EPSYLON EA 4556, F34000, Montpellier, France. Contact: Anne-marie.costalat@univ-montp3.fr.

2 Doctorant en Psychologie sociale et du travail à l'université Paul Valéry Montpellier 3. Adresse postale: Univ Paul Valéry Montpellier 3, EPSYLON EA 4556 F34000, Montpellier, France. Contact: Audrey.gosset@univ-montp3.fr 


\section{Identity dynamics and project}

The aim of this article is to present the processes that drive the phasic identity dynamics of social identity, allowing us to define simultaneously a representation of self and the personal and professional project. As a result, it implies differentiated levels of anchorages in the organizations, groups and society that contribute to shaping the self-project. We will illustrate this, thanks to cases and situations related to the context, autobiographical memory but also emotions, feeling of capacity and motivation. One of our orientations stipulates that social identity is organized in a representational dynamic in which the phases of action become facilitators of identity construction and leaving forms in experiential memory necessary for the construction of the project.

Keys words: Identity dynamics, project, action, orientation.

\section{Dinâmica de identidade e projeçáo}

O objetivo deste artigo é apresentar os processos que impulsionam a dinâmica de identidade física da identidade social, permitindo definir simultaneamente uma representação de si próprio e o projeto pessoal e profissional. Como resultado, implica níveis diferenciados de âncoras nas organizaçóes, grupos e sociedade que contribuem para moldar o autoprojeto. Vamos ilustrar isso, graças a casos e situaçôes relacionadas ao contexto, memória autobiográfica, mas também emoçóes, sensação de capacidade e motivação. Uma de nossas orientaçóes estipula que a identidade social é organizada em uma dinâmica de representação em que as fases de ação se tornam facilitadoras da construçâo da identidade e deixando formas na memória experiencial necessário para a construção do projeto.

Palavras-chave: Dinâmica da identidade, projeto, ação, orientação. 
La dynamique identitaire est activée par la représentation du «projet du soi» dans plusieurs sphères d'activités, c'est un système complexe où émergent des processus en interaction qui activent une dynamique représentationnelle de soi et de la société que l'on peut qualifier de dynamique identitaire. En effet, elle entraîne l'imbrication étroite des facteurs affectifs et cognitifs et du contexte (groupes, organisations, société), qui interagissent et qui constituent des régulations dynamiques pouvant être en relation avec l'élaboration de stratégies. Une identité qui s'organise dans le temps (continuité) à partir de "phases identitaires» plus ou moins claires. Des phases de dynamique représentationnelle où les phases d'action sont des expériences sociales et vivantes. Et où elles laissent ainsi des formes d'empreintes dans la mémoire autobiographique donnant à l'identité toute sa réalité expérientielle. L'expérience de l'action est mobilisée et mise en perspectives pour gérer un projet. En psychologie sociale on a souvent délaissé le pouvoir de l'action dans la dynamique identitaire, en se centrant sur la dimension représentationnelle et la conscience du soi. Or cet article a comme objectif de considérer l'aspect de la dynamique représentationnelle de soi, à partir du pouvoir de l'action qui apparait comme une dimension déterminante. On peut se référer à Nuttin (1980) dans son excellent ouvrage sur la motivation humaine, qui souligne l'importance de l'action dans l'orientation du projet ainsi que la capacité pour le réaliser. Nous considérons l'action comme un projet à temporalité moyenne, pour l'extraire d'un cadre behavioriste où l'action est simplement une exécution comportementale. Le fait que le sujet puisse parler de son action est largement évocateur. En effet, l'action est au cœur de la construction du sens, car elle peut être racontée et échangée puisqu'elle met en relation les aspirations, les représentations, mais aussi les valeurs et le sentiment de capacité régulant le système capacitaire. 


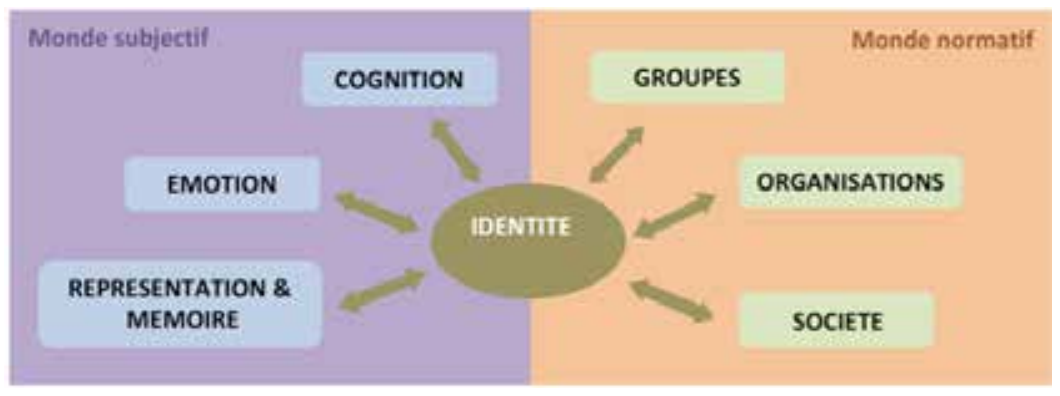

Figure 1. Dynamique identitaire (Costalat-Founeau, 2016)

\section{L'Action comme une régulation identitaire}

L'idée que l'action est une fonction identitaire n'est pas nouvelle. Goffman (1973) a participé à la conceptualisation du lien entre l'action et l'identité à partir de modalités qui permettent à une personne d'assumer différents rôles. Il met ainsi l'accent sur les aspects visibles de la personne et de son adhésion au rôle, ce qui permet au sujet de valoriser ses performances sociales (ses capacités) tel l'acteur qui essaie de ne pas dévier du rôle que l'on attend de lui. L'auteur considère que la vie sociale est une «vaste scène» où les acteurs sont amenés à respecter certains rituels, certaines lignes de conduite de la société. Généralement la présentation de soi est congruente avec l'image que le sujet désire donner de lui-même. Cet ordre normatif est bien conditionné par la capacité subjective, ce qui explique ses variations. Si l'identité est une présentation de soi qui se construit dans une dynamique de socialisation, l'action fait le lien à la fois pratique et communicationnel avec la société et procure une forme d'autonomie comme le souligne Auzoult (2010). Cette emprise sociale est aujourd'hui de façon déterminante accordée au sujet (Touraine \& Khosrokhavar, 2000). Laction a donc un pouvoir énergétique, on parle de "champs d'action» (Lewin, 1951), le réduisant à un milieu. Cette définition se centre essentiellement sur son aspect dynamique, énergétique et considère deux mondes juxtaposés: le sujet et l'environnement. Cette théorie volontariste de l'action (Blumer, 
1969) envisage ce dernier comme une construction fondée sur des buts, des sens, des intentions, et d'autres "états futurs». Elle peut être conçue comme une séquence intentionnelle et donne un sens à la fois au sujet individuel et collectif (Von Cranach, 1995). Enfin Bolstanski et Thévenot (1991) évoquent la notion de temporalité de surface qui correspond à une action séquentielle et une temporalité de fond qui définit les compétences de l'acteur se situant au niveau inconscient de l'action.

Par ailleurs, dans une approche de cognition sociale, Amerio (1995) définit l'action comme une compétence. Cette perspective est plus générale que la notion de compétence développée par Bandura (2003), plus restrictive, qui détaille précisément la manière dont le sentiment de compétence se développe. Ce sentiment est une attente d'efficacité personnelle, définie comme la croyance en sa propre capacité à organiser et à exécuter les séries d'actions requises pour produire certains résultats. Bien qu'il s'agisse à l'origine d'un sentiment personnel, la notion d'attente d'efficacité a été également appliquée au groupe. Elle correspond alors au jugement qu'un groupe possède sur ses capacités conjointes à organiser et à exécuter les séries d'actions requises pour produire des niveaux spécifiques de performance (Gernigon \& Ninot, 2005). Dans ses recherches sur l'efficacité du soi, Bandura (2003) met l'accent sur l'accessibilité des objectifs qui procurent au sujet satisfaction lorsque ceux-ci sont atteints et d'une certaine façon l'auteur montre l'influence implicite de l'estime de soi dans la mesure où les personnes cherchent une forme de réussite provoquant une émotion positive. On constate que ce modèle est traité dans l'attente d'une production de résultats, d'efficacité immédiate et d'un contrôle de l'environnement dans une perspective behavioriste et que la capacité subjective est laissée entre parenthèses. En réinterprétant ces résultats à partir du modèle capacitaire Costalat-Founeau (1997; 1999; 2008), on peut observer qu'au-delà du contrôle cognitif, il existe un besoin de reconnaissance de soi à la fois par soi et par autrui.

L'action n'est pas simplement réactive à l'environnement, elle met à disposition des moyens et des ressources propres afin que le 
sujet ajuste ses capacités. C'est donc le caractère normatif et cognitif de l'action et de la capacité que l'on voudrait souligner. Elle exerce une fonction constructive de l'identité, car elle projette le sujet et inscrit une expérience, et, par la fonction normative qui est incluse, elle contribue à donner des formes de validation temporaire qui sont autant d'encouragements. En effet, on a mis en évidence chez un groupe de cadres au chômage (Mary \& Costalat-Founeau, 2017) et d'infirmières (Guillen, 2005), des mécanismes de construction identitaire professionnelle qui passent par des ancrages sociaux, dans le groupes de stagiaires pour la première recherche, ou encore au travers du groupe des malades pour la seconde étude. En ce qui concerne les formateurs, les groupes qu'ils accompagnent, de façon limitée dans le temps (celui de la durée de la formation est de six mois) leur permettent de construire une identité professionnelle virtuelle dans une société en changement. De ce fait, au cœur de la crise et souvent avec des publics en situation d'échec, ces formateurs trouvent des réponses qui leur sont propres et des moyens d'innovation qui légitiment leurs capacités face au groupe et à l'institution. Ce nouvel espace de reconnaissance sert de cadre de référence, de champs de comparaison sociale. On peut ici évoquer le concept d'identité virtuelle développé par Dubar (1991) et Sainseaulieu (1998) à propos des chômeurs en formation. Dans sa quête identitaire professionnelle, l'individu cherche et trouve la «validation sociale et la reconnaissance». Ceci facilite un investissement socioaffectif qui permet d'élaborer une forme d'objectivation "construite» et «limitée» dans le temps qui donne un sens à l'action. La plasticité supposée dans le modèle de compétence des acteurs implique des séquences courtes d'action. Et la capacité, en relation avec l'action, passe par le pouvoir du sujet acteur, qui gère et auto-organise, ajuste, des champs de références plus ou moins normatifs afin de permettre une confrontation identitaire (Martinez \& Costalat-Founeau, 2005). Le sentiment de capacité est au cour de leur subjectivité et peut compléter le besoin de reconnaissance développé par Codol (1986) qui est davantage lié et soumis à la désirabilité sociale et par conséquent à l'influence sociale, même s'il s'en défend. Nous présenterons par la 
suite, le modèle capacitaire qui permet d'intégrer à la fois la dimension subjective et normative.

\section{Capacité et émotion}

Nous pensons que le sujet est à la fois auteur, acteur et observateur dans l'environnement. Ainsi de notre point de vue, cette réalité n'est pas stable ni prescrite. Le sujet participe à sa construction et interagit avec. Il gère l'environnement à partir de ses capacités dans le souci permanent de les concrétiser, ce qui lui procure satisfaction, plaisir et par conséquent une mobilisation émotionnelle positive. Ces effets capacitaires activent des tonalités affectives, positives ou négatives, qui favorisent une forme de clairvoyance, une perspicacité à juger les choses avec exactitude et un pouvoir de contrôle de l'environnement, mais aussi des capacités dont on dispose.

La clairvoyance (acuité représentationnelle) caractérise la première situation. Dans la seconde, plus diffuse, le sujet se laisse davantage déborder par les émotions, se sent inefficace et l'on peut rencontrer des phénomènes d'alexithymie qui conditionnent le retrait. Nous caractérisons ces effets comme des effets capacitaires, d'une part la capacité subjective (je peux), qui confère au sujet une capacité à agir dans le sens où il maîtrise les enjeux, les moyens et définit les objectifs, et d'autre part une capacité normative, liée à la validation sociale et qui entraîne des formes d'approbation ou de désapprobation sociales. Ces capacités sont étroitement interdépendantes dans le sens défini par Huteau (1985) et constituent le système capacitaire qui a un rôle essentiel dans l'intention, la concrétisation et la finalité de l'action.

Les réactions affectives qui accompagnent l'activité autorégulatrice orientée vers les buts sont étroitement enracinées dans les croyances relatives aux probabilités de succès et dans les croyances en matière d'efficacité personnelle (Gegas, 1989). Le fait de croire à la compétence personnelle et le progrès vers les buts sont des déterminants puissants des émotions qui peuvent alors influencer l'autorégulation et par voie 
de conséquence la dynamique identitaire. C'est dans une dynamique des capacités (subjectives et normatives) que s'opèrent les phases identitaires et, selon les consonances ou les dissonances du système capacitaire, des phases plus ou moins claires ou diffuses s'alterneront (Costalat-Founeau, 1999).

Nous allons présenter deux exemples explorés à partir de l'egoécologie et le projet de soi. Le premier celui de la bibliothécaire, le second celui des demandeurs d'emploi.

L'ego-écologie (Zavalloni, 2007) permet de repérer, grâce à la méthodologie de l'investigateur multistade de l'identité sociale (IMIS), des phases identitaires qui sont des «moments-forces» de la subjectivité. Elle permet aussi de montrer les différentes cohérences entre le projet, l'action et le sentiment de capacité. Les différentes consonances et dissonances entre la capacité subjective et objective peuvent être repérées à travers l'analyse des relations entre Soi et Alter et peuvent ainsi représenter un support pour les applications de l'investigation d'un projet (Costalat-Founeau, Klimekova \& Blanc, 2003). Précisément dans cette première recherche on a pu montrer que le métier de bibliothécaire, choisi dans le premier temps par Isabelle dans une démarche d'accompagnement pour l'emploi, n'était pas en cohérence avec ses valeurs, ses désirs et ses capacités. Ce choix n’a été qu'une solution pour assumer sa responsabilité de mère de famille. Il s'agissait d'une solution de repli, lui permettant de faire le deuil d'une profession artistique qui lui semblait ne pas pouvoir s'articuler avec une nécessité de subvenir aux besoins financiers de son foyer. En revanche, le choix de la formation d'administrateur de spectacle lui a permis, d'une part de surmonter le sentiment de dépendance financière à l'égard de son conjoint et de mieux gérer ses angoisses liées aux responsabilités de mère, et, d'autre part de diminuer l'impact de la famille qui lui confirmait l'impossibilité de se réaliser dans un métier artistique. Le maintien chez le sujet d'une cohérence entre les capacités à la fois normatives et subjectives est une des conditions de l'émergence et de l'actualisation du projet. 
Par ailleurs, dans une étude comparative entre une situation d'accompagnement et de non-accompagnement, Gosset, CostalatFouneau, Faurie et Misantrope (2016) ont confirmé que l'aide à l'insertion professionnelle joue un rôle important dans l'appui et l'autonomie des individus en recherche d'emploi (Koen, Klehe $\&$ Van Vianen, 2013). Ici, l'accompagnement consent à une reconstruction du sentiment d'appartenance à un groupe avec un but commun, en permettant d'une part de rompre l'isolement du sujet et d'autre part, d'activer leur système capacitaire. Dans la situation de nonaccompagnement, face à cette dévalorisation, la perte de confiance en soi entraîne le sujet dans une attitude de résignation et d'incapacité. En comparaison, l'accompagnement psychosocial ayant pour objectif la formation d'un projet professionnel peut contribuer à uneévolution de sa dynamique identitaire (Eden \& Aviram, 1993). En effet, l'appartenance au groupe d'accompagnement active, chez le demandeur d'emploi, un lien d'appartenance actif, avec un objectif commun, comme nous le précise le participant accompagné: «ici on est écouté, on est en groupe et tous dans la même situation et puis c'est différentı. L'individu semble moins s'identifier au groupe des demandeurs d'emploi, mais au groupe de personnes travaillant sur leur projet professionnel, une catégorie socialement valorisée (Tajfel \& Turner, 1979), une dynamique de groupe peut se créer, bénéficiant alors à l'ensemble des participants. La construction du projet professionnel viable dans le temps est un indicateur essentiel de la confiance que le sujet a de ses capacités, à mobiliser ses compétences vers un but précis (Chaix, 1994).

Les deux schémas ci-dessous illustrent ces processus après avoir réalisé la passation de l'IMIS.

1. Le premier démontre les processus identitaires émergents avec le soutien social et formatif: La motivation et l'autonomie. Ici, nous voyons que la construction d'un projet professionnel a joué un rôle déterminant dans l'activation de la dynamique identitaire. La théorie de l'action (Young \& Valach, 2006) relate bien de ce phénomène dans le sens où le projet et l'action forment des processus permettant d'organiser les comportements individuels dans un but précis. Ils 
sont tous deux la clé du fonctionnement humain puisque sans projet l'individu ne peut subsister (Boutinet, 1990). C'est d'ailleurs, ce que démontre aussi Welnowki-Michelet (2008) en mettant en évidence l'importance d'un environnement porteur afin de placer le demandeur d'emploi dans une démarche de recherche-action lui permettant de renforcer son sentiment capacitaire positif.

2. Le deuxième schéma quant à lui renvoie aux processus identitaires des chômeurs sans soutien social: La résignation et l'inaction. Les individus ressentent une insatisfaction générale face aux évènements de la vie au regard d'un sentiment de "non-appartenance professionnelle» (Desmette, Liénard \& Dalla-Valle, 2007). Ceci étant marqué par une identité fragilisée et l'absence d'une vision claire de leur avenir professionnel. Les sujets ont un sentiment capacitaire négatif les entrainant dans une phase de diffusion où l'engagement dans l'action est difficile, voire impossible, puisque le sujet est submergé par ses émotions. Par ailleurs, on observe également une production d'émotions négatives plus marquée chez les sujets non accompagnés (Sheeran, Abrams \& Orbell, 1995), caractérisée par une peur quant à l'avenir et une résignation plus importante face aux évènements qui leur arrivent.

\section{Conclusion}

Les exemples présentés illustrent bien la manière dont l'intériorisation active de l'environnement social sert de base à la construction de l'identité dans une interaction en définissant les projets. La construction de nouveaux projets implique donc une remise en question de l'identité à travers le sens donné à nos actions, ainsi que nos modes de rapports aux autres et à soi.

On peut conclure en stipulant que l'identité n'est pas seulement conscience de soi et appartenance groupale: elle est aussi mise "en acte», car elle implique des formes temporaires de cohérence et stabilise le système capacitaire. On observe aussi que ces formes de cohérences 

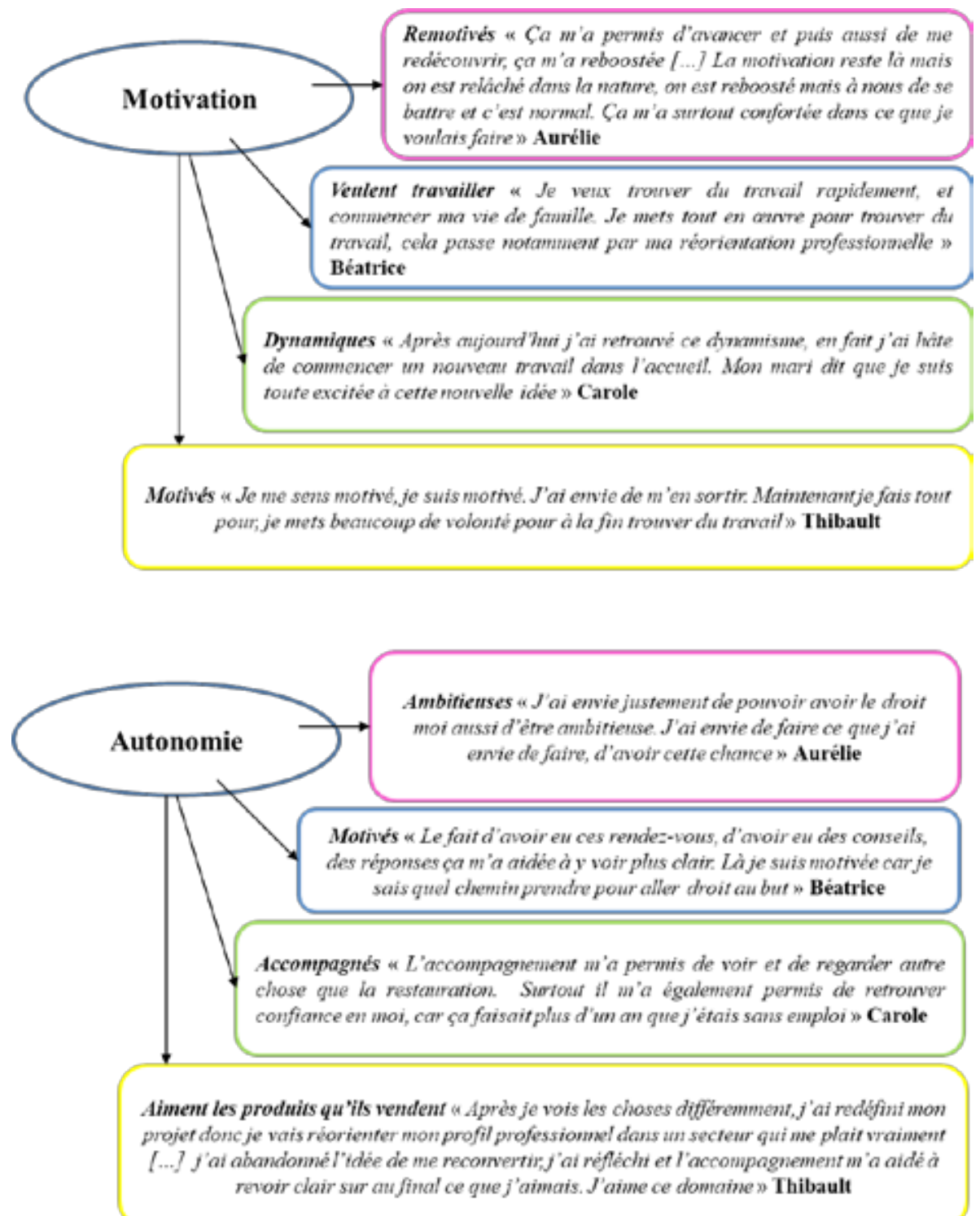

Figure 2. Phase d'acuité représentationnelle 

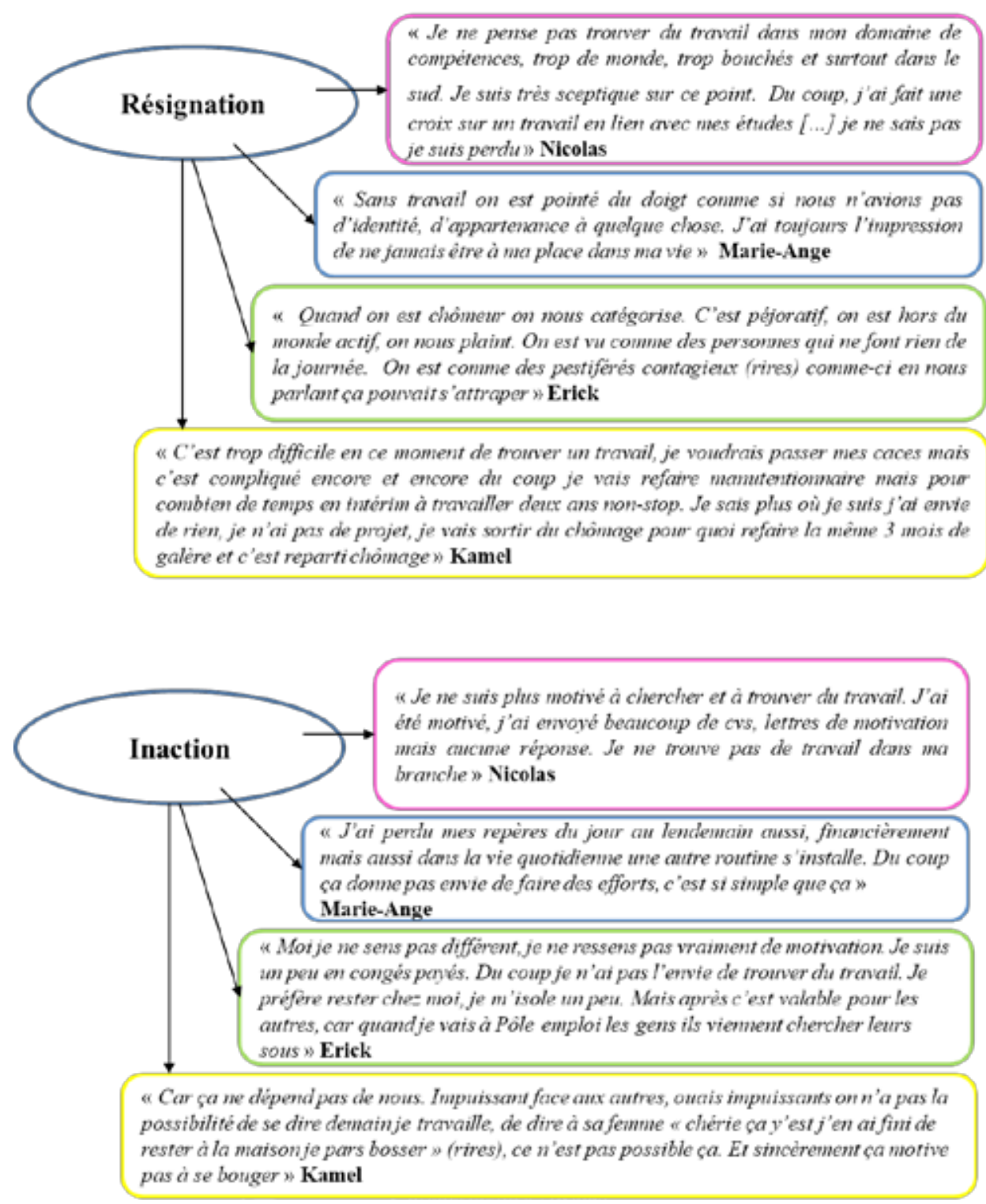

Figure 3. Phase de diffusion représentationnelle 
sont difficiles à établir, car les crises sociales et les contextes engendrent des mouvements complexes ainsi que des incertitudes. Le sujet est alors bien obligé de changer, de déplacer ou de construire des espaces de référence pour accomplir de nouveaux projets et pour rééquilibrer son système capacitaire que nous considérons comme un vecteur central de l'auto-régulation de la dynamique identitaire. Or, on peut constater que la créativité de l'action efface l'influence normative comme le souligne (Joas, 1999) dans la créativité de l'agir. Nous avons ici un bel exemple avec les formes identitaires qu'offrent les métiers de la création (Garcia de la Barga, 2015).

Ceci nous conduit à penser que la construction de l'identité s'établit dans une dynamique phasique certes grâce à des modes de reconnaissance sociale, et que la «capacité d'action» devient un vecteur force de développement et de créativité.

\section{Références}

Adorno, T.W. (2007). Rendre compte de soin. In Judith Bulter (Ed.), Le récit de soi. Paris: Presses Universitaires de France.

Amerio, P. (1995). Fondamentti Thorici di psicologia sociale, Il Molino. Auzoult, L. (2010). Validation d'une échelle de mesure de la situnomie-autonomie. L'orientation scolaire et professionnelle, 39(2), 197-217.

Bandura A. (2003). Auto efficacité: Un sentiment d'efficacité personnelle. Paris: De Boeck.

Blumer, H. (1969) Symbolic Interactionism: Perspective and Method. Englewood Cliffs, NJ: Prentice- Hall.

Bolstanski, L. \& Thévenot, L. (1991). De la justification. Les économies de la grandeur. Paris: Gallimard.

Boutinet, J.P. (1990). Anthropologie du projet. Paris: Presses Universitaire de France.

Butler, J. (2007). Le récit de soi. Paris: Presses Universitaires de France. 
Chaix, M.C. (1994). Des conditions pour apprendre dans les dispositifs de formation école-entreprise. Éducation permanente, (119), 164-165.

Codol, J.P. (1986). Estimation et expression de la ressemblance et de la différence entre pairs. L'année psychologique, 86(4), 527-550. https://doi.org/10.3406/psy.1986.29169

Costalat-Founeau A.M. (1997). Identité Sociale et Dynamique Représentationnelle. Rennes: Presses Universitaires de Rennes.

Costalat-Founeau A.M. (1999). Identity dynamic, action and context. Journal for the theory of social behaviour, 29(3), 289-300. https:// doi.org/10.1111/1468-5914.00103

Costalat-Founeau, A. (2008). Identité, action et subjectivité: Le sentiment de capacité comme un régulateur des phases identitaires. Connexions, 1(89), 63-74. https://doi.org/10.3917/ cnx.089.0063

Costalat-Founeau, A-M. (2016). Identité professionnelle. In G. Valéry, M-E. Bobillier-Chaumon, E. Brangier \& M. Dubois (Eds.), Psychologie du travail et des organisations, 110 notions clés, 236-239. Paris: Dunod.

Costalat-Founeau A.M., Klimekova M. \& Blanc, C. (2003). Ego-ecology and professionnal project: A study in identity construction. In A-V Rigas (Ed.), Social Clinical Psychology: Theory, Methodology, and interventions, 233-251. Athens: Ellinika Grammata.

Desmette, D., Liénard, G. \& Dalla Valle, C. (2008). Les activités d'insertion sociale: occupation ou insertion? Dans G. Herman (Ed.), Travail, chômage et stigmatisation, 253-281. Bruxelles: De Boeck.

Dosse, F. (1995). L'empire du sens. Paris: la découverte.

Dubar, C. (1991). La Socialisation: Construction des identités sociales et professionnelles. Paris: Armand Colin.

Eden, D. \& Aviram, A. (1993). Self-efficacy training to speed reemployment: Helping people to help themselves. Journal of applied Psychology, 78 (3), 352-360. https://dx.doi. org/10.1037/0021-9010.78.3.352 
Garcia de La Barga, C. (2015). Dynamique identitaire et capacitaire en situation de formation: le cas des créateurs de mode. Thèse, Université Paul Valéry-Montpellier III.

Gegas, V. (1989). The social psychology of self-efficacy. Annual Review of Sociology, 15(1), 291-316. https://doi.org/10.1146/annurev. so. 15.080189 .001451

Gernigon, C., Ninot, G. (2005). La compétence: un sentiment personnel. In J.-L. Ubaldi (Ed.), Les compétences, 45-62. Paris: Revue EPS.

Goffman E. (1973). La mise en scène de la vie quotidienne. Paris: éditions de minuit.

Gosset, A., Costalat-Founeau, A.M., Faurie, I. \& Misantrope, Y. (2016). Dynamique Identitaire et insertion professionnelle: le cas de demandeurs d'emploi de longue durée, Bulletin de psychologie. In press.

Guillen, S. (2005). Identité et sentiment capacitaire professionnel: une étude réalisée auprès des infirmières. In A-M. Costalat-Founeau (Ed.) Identité sociale et ego-écologie: théorie et pratique, 151-177. Paris: Sides.

Joas H. (1999). La créativité de l'agir. Paris: Cerf.

Huteau, M. (1985) Les conceptions cognitives de la personnalité. Paris: Presses Universitaires de France.

Koen, J., Klehe, U. C. \& Van Vianen, A. E. (2013). Employability among the long- term unemployed: A futile quest or worth the effort? Journal of Vocational Behavior, 82 (1), 37-48. https://doi. org/10.1016/j.jvb.2012.11.001

Lewin, K. (1951). Field theory in social science: selected theoretical papers. New York: Harper and Row.

Martinez, N. \& Costalat-Founeau, A.-M. (2005). Comparaison de deux situations de réussite confrontée et normalisée chez des lycéennes d'origine culturelle différente. Connexions, 1 (83), 142-164. https://doi.org/10.3917/cnx.083.0149

Mary, G. \& Costalat-Founeau, A.M. (2017). Investigating the professional identity dynamic in career counselling: The 
socioconstructivist interview. International journal for educationalandvocational, 1-20. http://doi.org/10.1007/s10775017-9350-5

Nuttin, J. (1980). Théorie de la motivation humaine. Du besoin au projet d'action. Paris: Presses Universitaires de France.

Palmade, J. (2003). L'incertitude comme norme. Paris: Presses Universitaires de France.

Ricour, P. (2004). Parcours de la reconnaissance. Paris: Stock.

Sainseaulieu, R. (1998). L'identité au travail d'hier à aujourd'hui. L'orientation scolaire et professionnelle, 27(1), 77-93.

Sheeran, P., Abrams, D. \& Orbell, S. (1995). Unemployment, selfesteem, and depression: A social comparison theory approach. Basic and applied social psychology, 17, 1-2, 65-82. https:// dx.doi.org/10.1080/01973533.1995.9646132

Tajfel, H. \& Turner, J.C. (1979). An integrative theory of intergroup conflict. In W.G. Austin \& S. Worchel (Eds.), The social psychology of intergroup relation. Monterey, CA: Brooks/Cole.

Touraine, A. \& Khosrokhavar, F. (2000). La recherche de soi. Dialogue sur le sujet. Paris: Fayard.

Von Cranach, M. (1995). Social representations and individual actions: Misunderstandings, omissions and different premises. Journal for the Theory of Social Behviour, 24(3), 285-293.

Welnowski-Michelet, P. (2008). L'identité à l'épreuve de l'exclusion socioprofessionnelle. Paris: L'Harmattan.

Young, R.A \& Valach, L. (2006). La notion de projet en psychologie de l'orientation. L'orientation scolaire et professionnelle, 35(4), 495-509.

Zavalloni, M. (2007). Ego-écologie et identité: une approche naturaliste. Paris: Presses Universitaires de France.

Recibido: 13 de septiembre, 2017

Revisado: 30 de octubre, 2017 Aceptado: 02 de noviembre, 2017 